\title{
Efeito anti-helmíntico de taninos condensados em nematódeos gastrintestinais de ovinos (Ovis aries)
}

\author{
Anthelmintic effect of condensed tannins in gastrointestinal \\ nematodes of sheep (Ovis aries)
}

\author{
Eidi Yoshihara ${ }^{1 *}$; Alessandro Pelegrine Minho²; Milton Hissashi Yamamura ${ }^{3}$
}

\section{Resumo}

\begin{abstract}
As helmintoses gastrintestinais constituem um dos principais fatores limitantes na produção de ovinos em todo o mundo. O desenvolvimento da resistência dos parasitos aos anti-helmínticos tem gerado grandes perdas na produção de pequenos ruminantes. Na busca por novas alternativas de controle efetivo de nematódeos gastrintestinais em pequenos ruminantes, a fitoterapia constitui um campo promissor de pesquisas na tentativa de reduzir a utilização de tratamentos químicos, visando uma produção agroecológica. Muitas plantas desempenham um papel crucial na manutenção da vida animal e humana. Durante a evolução humana muitas interações com outros organismos foram estabelecidas com consequências para ambas as partes de diversas maneiras. Plantas desenvolveram mecanismos bioquímicos para se defender de antagonistas biológicos que atuam como seus inimigos naturais. Este princípio tem levado os cientistas a procurar compostos bioativos produzidos pelas plantas contra os agentes patogénicos. Uma vez identificados, estes metabólitos são avaliados em relação a seu efeito sobre doenças de importância não só para a saúde pública, mas também na produção animal. O presente artigo apresenta uma revisão dos estudos realizados com plantas taniníferas cientificamente testadas em todo o mundo para o controle das parasitoses gastrintestinais em ruminantes.
\end{abstract}

Palavras-chave: Fitoterapia, helmintos gastrintestinais, pequenos ruminantes, taninos

\begin{abstract}
Gastrointestinal helminthiasis infections are considerate one of the main limiting factors in sheep production worldwide. The development of parasite resistance to anthelmintics has caused significant losses of small ruminant production. In the search for new alternatives for effective control of gastrointestinal nematodes in small ruminants, phytotherapy is a promising field of research in an attempt to reduce the usage of chemical treatments, aimed at ecological production. Many plants play a crucial role in maintaining animal and human life. During human evolution many interactions with other organisms were established and they have affected each other in many ways. Plants have developed biochemical mechanisms to defend themselves from biological antagonists that act as their natural enemies. This principle has led scientists to search for bio-active compounds produced by plants against pathogens. Since these discovers their metabolites are evaluated against diseases of importance not only in public health, but also in animal production. This article presents a review of studies with tanniniferous plants scientifically tested worldwide for the control of gastrointestinal parasites in ruminants.
\end{abstract}

Key words: Phytotherapy, helminthiasis, small ruminants, tannins

\footnotetext{
${ }^{1}$ Pesquisador Científico da Agência Paulista de Tecnologia dos Agronegócios, Polo Alta Sorocabana, Presidente Prudente, SP. E-mail: eidi@apta.sp.gov.br

${ }^{2}$ Pesquisador A, Embrapa Pecuária Sul, Bagé, RS. E-mail: alessandro.minho@embrapa.br

${ }^{3}$ Prof. Dr. do Dept ${ }^{\mathrm{o}}$ de Medicina Veterinária Preventiva, Centro de Ciências Agrárias, Universidade Estadual de Londrina, UEL, Londrina, PR. E-mail: yamamura@uel.br

* Autor para correspondência
} 


\section{Parasitismo em ovinos}

Um dos principais fatores limitantes encontrados na ovinocultura são os endoparasitas, sendo os nematódeos gastrintestinais (NGI) os de maior importância. Dentre os NGI destacamse Haemonchus contortus, Trichostrongylus colubriformis, Strongyloides spp.,Cooperia curticei e Oesophagostomum columbianum (AMARANTE et al., 2004). Os ovinos apresentam infestações helmínticas em todas as faixas etárias e os cordeiros desmamados constituem a categoria etária mais acometida pela verminose (ECHEVARRIA; PINHEIRO; CORRÊA, 1989), acarretando não somente atraso no desenvolvimento corporal, mas também na produção e qualidade da carne, lã e, até mesmo, aumento da mortalidade dos animais jovens (MOLENTO; PRICHARD, 1999).

Os helmintos acarretam altas perdas econômicas, seja em criações extensivas (KOHLER, 2001) ou intensivas como observado por Niezen et al. (1998) na Nova Zelândia, que consideram os parasitos gastrintestinais o maior impedimento ao aumento da taxa de crescimento animal. Entretanto, segundo Amarante (2001) não se pode relacionar parasitismo com doença, visto que grande parte dos animais infectados de uma propriedade encontra-se em boas condições de saúde. A capacidade de apresentar boas condições está relacionado à capacidade imunológica do hospedeiro em manter o número de parasitos gastrintestinais em níveis aceitáveis, e quando isso ocorre pode-se afirmar que a relação parasito-hospedeiro está em equilíbrio.

O H. contortus é o parasito mais patogênico para pequenos ruminantes em regiões tropicais e subtropicais em todo o mundo, inclusive no Brasil. O parasito adulto é hematófago, causando problemas gástricos e perdas de proteínas séricas, tendo seu efeito agravado pela anemia (STRAIN; STEAR, 2001). Nos países tropicais, as infecções causadas por $T$. colubriformis também são consideradas de grande importância na criação de ovinos, logo após a hemoncose. Mesmo as infecções parasitárias consideradas por alguns autores como subclínicas, diminuem o ganho de peso, o consumo voluntário, a produção de leite e lã, podendo ainda, prejudicar a deposição de tecidos moles e o crescimento esquelético dos animais (BUTTER et al., 2000).

$\mathrm{O}$ ciclo evolutivo dos nematoides trichostrongilideos (Família Trichostrongylidae) é semelhante e envolve uma fase de vida-livre no ambiente e outra parasitária, no hospedeiro. A fase de vida livre tem início com a eliminação dos ovos nas fezes dos animais contaminados para o ambiente. No ambiente, a larva se desenvolve dentro do ovo e é liberada após a eclosão. A larva cresce e sofre duas ecdises antes de se tornar infectante $\left(\mathrm{L}_{3}\right)$ fase na qual a larva mantem duas bainhas (aumentando sua sobrevida no ambiente), então migra do interior do bolo fecal para as pastagens ao redor. A evolução da larva até a forma infectante geralmente se dá dentro de 5 a 7 dias. A fase de vida parasitária tem início com a ingestão da $\mathrm{L}_{3}$, a qual se desenvolve até L4 e L5, essa fase se completa com a presença do parasito adulto no trato digestório. A reprodução desses parasitas se dá de forma sexuada com eliminação de ovos nas fezes do hospedeiro e início de novo ciclo.

\section{Controle do parasitismo gastrintestinal e resistência}

O controle parasitário dos nematódeos gastrintestinais é baseado em tratamentos repetidos do animal com drogas anti-helmínticas (AMARANTE et al., 1997). Segundo TorresAcosta e Hoste (2008) para o controle de NGI dois pontos primordiais devem ser abordados: (i) nos hospedeiros, melhorando a resistência, a resiliência e a redução da carga parasitária; (ii) no ambiente, com a redução da contaminação das pastagens por $\mathrm{L}_{3}$.

A prática de tratamentos repetidos favorece o surgimento de populações de helmintos resistentes às drogas existentes (TORRES-ACOSTA; HOSTE, 
2008). Segundo Sangster e Gill (1999) a resistência aos anti-helmínticos é o declínio da eficiência de uma droga contra uma população de parasitos, anteriormente suscetível àquele tratamento. A resistência contra todos os grupos de fármacos utilizados é uma realidade mundial (MOLENTO; PRICHARD, 1999), sendo um importante problema, principalmente em ovinos e caprinos (GOPAL; POMROY; WEST, 1999).

O primeiro relato de resistência a anti-helmínticos para controle de nematódeos gastrintestinais de ovinos foi descrito na década de 1960, após avaliação de tratamentos com tiabendazole (DRUDGE et al., 1964). Este problema disseminou-se pelo mundo inteiro e perpetua-se até os dias atuais. Em pesquisa realizada por Bartley et al. (2003) em 90 propriedades da Escócia, os autores detectaram que $64 \%$ das propriedades apresentaram resistência ao tiabendazole. No Quênia, Waruiru (1997) avaliou a eficácia do closantel, albendazole e levamisole em ovinos e relatou a presença da resistência múltipla de $H$. contortus a todas as drogas testadas.

O primeiro caso de resistência de nematódeos gastrintestinais para lactonas macrocíclicas em pequenos ruminantes na Suíça, foi relatado por Schnyder et al. (2005), onde encontraram isolados de $H$. contortus resistentes à ivermectina. Em estudo realizado na Nova Zelândia, Southerland et al. (2008) encontraram isolados de Teladorsagia, Trichostrongylus e Haemonchus resistentes à ivermectina, relatando o primeiro caso de resistência à ivermectina em T. colubriformis. Na Espanha, Alvarez-Sanchez et al. (2006) relataram a presença da resistência para benzimidazois, imidazotiazoles e lactonas macrocíclicas.

Na Argentina, Fiel et al. (2011) avaliaram a eficácia dos anti-helmínticos, triclorfon, ivermectina, febendazole, levamisole e o closantel e encontraram resistência múltipla aos produtos testados, uma vez que não foi relatada redução na contagem de ovos por grama de fezes (OPG). George et al. (2011), avaliaram a eficácia de anti-helmínticos em diferentes estados de Trinidad, concluíram que os nematódeos desenvolveram resistência para albendazole, fenbendazole e levamisole, fato esse não observado para ivermectina.

No Brasil, após o primeiro relato de resistência aos anti-helmínticos em ovinos no Rio Grande do Sul (DOS SANTOS; GONÇALVES, 1967), não faltaram relatos de isolados resistentes aos fármacos. Echevarria, Pinheiro e Corrêa (1989) examinando rebanhos no município de Bagé-RS encontraram rebanhos com helmintos resistentes aos benzimidazois, ao tetramisole e rebanhos com resistência múltipla. Estudo realizado no Rio Grande do Sul por Cezar et al. (2010) demostrou que nos testes de eficácia anti-helmíntica não ocorreu a redução na contagem de $\mathrm{OPG}$, concluindo então a presença da resistência a levamisole, moxidectina, albendazole, ivermectina, nitroxil, disofenol, triclorfon, closantel e combinação de ivermectina + levamisole + albendazole no Estado do Rio Grande do Sul.

Ramos et al. (2002) avaliaram a resistência em propriedades do Estado de Santa Catarina e relataram a presença de resistência a ivermectina, ao albendazole, ao closantel e ao levamisole. De acordo com os autores, a multirresistência está presente na maioria dos rebanhos. No Paraná, Souza e Thomaz-Soccol (1997) verificaram que rebanhos ovinos apresentaram resistência anti-helmíntica ao oxfendazol, levamisole, tetramisol, ivermectina, moxidectina, closantel e as associações tetramisol + disofenol e oxfendazol + closantel. Em outro trabalho, também no Paraná, Thomaz-Soccol et al. (2004), avaliaram a eficiência dos anti-helmínticos em diferentes propriedades e também verificaram que a prevalência de resistência foi alta para todos os anti-helmínticos avaliados, onde relataram que todas as propriedades apresentaram resistência múltipla.

A resistência múltipla no Estado de São Paulo foi avaliada por Almeida et al. (2010), quando testaram os produtos químicos moxidectina, closantel, 
triclorfon, fosfato de levamisole, albendazole e ivermectina, concluindo que isolados de $H$. contortus e T. colubriformis apresentam múltipla resistência a todas as drogas testadas. SczesnyMoraes et al. (2010), avaliaram a eficácia antihelmíntica em propriedades produtoras de ovinos no Estado do Mato Grosso do Sul e concluíram que a resistência aos fármacos albendazole, closantel, ivermectina, levamisole, moxidectina, triclorfon e associação albendazole + ivermectina + levamisole está instalada na maioria dos rebanhos do Estado de Mato Grosso do Sul.

O desenvolvimento de isolados de parasitos resistentes às várias classes de anti-helmínticos é um fenômeno comum em muitos países e identificado em muitas espécies de nematódeos (KAPLAN, 2004), atingido níveis que inviabilizam a criação de ovinos em algumas regiões do hemisfério Sul (JACKSON; COOP, 2000). Além disso, os antihelmínticos disponíveis no mercado possuem algumas limitações, tais como alto custo, presença de resíduos nos alimentos, riscos de poluição ambiental e redução na produção de ovinos, devido a sua baixa eficácia (WALLER, 1997). Na busca de novas alternativas de controle efetivo dos NGI em pequenos ruminantes, vários pesquisadores têm se empenhado em testar plantas com compostos bioativos que possam ser empregados no manejo integrado de parasitoses, mitigando os efeitos deletérios ao meio ambiente.

\section{Plantas com atividade anti-helmíntica}

Em todo o mundo, é crescente na medicina, o número de pesquisas com fitoterápicos que apresentam atividade contra vírus, bactérias, fungos e parasitos, não sendo diferente na medicina veterinária, onde pesquisas com plantas medicinais objetivam a redução de problemas sanitários no controle de várias doenças que comprometem a produtividade dos animais (NIEZEN et al., 1996). No Brasil, plantas medicinais são largamente utilizadas tanto nas áreas rurais como urbanas. As plantas são utilizadas em formulações de remédios caseiros como chás, xaropes, pós, ou com o desenvolvimento da indústria farmacêutica, em cápsulas e pílulas (MATOS, 1997).

Várias causas levam ao aumento da procura por produtos alternativos, entre elas destacam-se o uso incorreto e/ou abusivo das drogas sintéticas, que pode desencadear reações adversas. Além disso, uma grande parte da população mundial não tem acesso ao tratamento farmacológico convencional (RATES, 2001).

Além da busca por medicamentos fitoterápicos e da tendência de produção orgânica, existe a problemática da resistência apresentada pelos nematódeos aos anti-helmínticos disponíveis em todo o mundo (MELO et al., 2003). Diante destes riscos, as plantas apresentam-se como uma alternativa natural, visto que muitas possuem mecanismos de defesa contra o ataque de patógenos, os quais são baseados na produção de compostos bioativos específicos que lhes conferem relativa resistência (BAR-NUM; MAYER, 1990).

A fitoterapia em medicina veterinária tem sido indicada, principalmente, para reduzir os custos dos tratamentos químicos e prolongar a vida útil dos produtos anti-helmínticos disponíveis no mercado, pois diminui a pressão de seleção sobre os isolados de nematódeos (VIEIRA et al., 1999). Os extratos brutos obtidos têm largo uso após a constatação de sua eficácia em testes de segurança e em ensaios biológicos. Quando a natureza química dos compostos envolvidos é conhecida, os métodos de extração do material podem ser baseados no uso da planta na medicina popular ou extrações com solventes de polaridades crescentes (RATES, 2001).

Os primeiros testes para avaliação da propriedade anti-helmíntica de uma planta medicinal são feitos in vitro, devido à facilidade de execução, baixo custo e rapidez em relação aos testes in vivo. As plantas ou seus compostos são colocados diretamente em contato com os diferentes estágios de desenvolvimento do parasito, afim de avaliar seu 
efeito sobre a eclosão de ovos, desenvolvimento larval e motilidade de larvas e adultos. É essencial que os primeiros testes sejam direcionados contra os helmintos de maior importância econômica (HAMMOND; FIELDING; BISHOP, 1997). Dentre os testes in vitro, aqueles que podem ser usados para avaliar os efeitos anti-helmínticos de uma planta são: teste de inibição da eclosão de ovos (IEO), teste de inibição da migração larval (IML), teste de inibição da alimentação larval (IAL), teste de desembainhamento (TD), teste de desenvolvimento larval (TDL) e teste de motilidade de adultos (TMA) (JACKSON; HOSTE, 2010). De acordo com os autores, para as primeiras análises de uma planta podem ser realizados os testes IEL, IML E IAL devido a sua praticidade.

Apesar de muitas plantas já terem sido descritas como possuidoras de atividade anti-helmíntica, poucas foram avaliadas cientificamente. Algumas pesquisas científicas conduzidas com diferentes espécies vegetais demonstram propriedades antiparasitárias em ruminantes (NIEZEN et al., 1995; ATHANASIADOU et al., 2001; HOSTE et al., 2006; MINHO et al., 2008a; MANOLARAKI et al., 2010; ALONSO-DÍAZ et al., 2011; OLIVEIRA et al., 2011, VON SON-DE FERNEX et al., 2012).

Alonso Díaz et al. (2010) relatam que é necessária a definição de muitos aspectos envolvidos tais como a parte da planta, idade e estado fisiológico da planta, aspectos relacionados à extração e dose adequada. Fatores ligados ao animal (espécie, idade, estado fisiológico) também devem ser considerados para que o mesmo possa ser utilizado no controle de nematódeos. É importante salientar que tanto os efeitos benéficos quanto os efeitos deletérios acarretados pelos taninos dependem de muitos fatores, sobretudo da concentração e da estrutura desses compostos (HOSTE et al., 2006).

\section{Taninos condensados}

De acordo com Niezen et al. (1995) o uso de plantas ricas em taninos condensados (TC) pode ser indicado como uma alternativa no controle de helmintos em ovinos, reduzindo o uso de produtos químicos, assim como os custos de produção. Portanto, além do melhoramento do rebanho, a utilização de fontes de TC na dieta dos animais pode diminuir a pressão de seleção sobre os NGI.

Diversos estudos confirmam que plantas têm sido utilizadas para o controle de NGI em pequenos ruminantes, fazendo uso de extratos, folhas, frutos, ou sementes oriundas de diferentes regiões (latitudes) do mundo e obtidas com diferentes técnicas (MAKKAR; FRANCIS; BECKER, 2007).

Os taninos compreendem um grande grupo de compostos encontrados principalmente em frutos verdes e plantas da família Leguminosae, muito disseminadas no reino vegetal. Esses compostos fenólicos são classificados conforme sua estrutura molecular em taninos hidrolisáveis ou taninos condensados, sendo os condensados também conhecidos como proantocianidinas. Os TC são os taninos mais comumente encontrados em plantas forrageiras, árvores e arbustos (BARRY; McNABB, 1999).

Duas hipóteses tem sido sugeridas para explicar o efeito anti-helmíntico dos TC contra uma população de nematódeos gastrintestinais. A primeira é o efeito direto, definido como a capacidade que os taninos têm de agir no parasito, afetando severamente os processos biológicos dos nematódeos (HOSTE et al., 2006). A segunda hipótese poderia ser o efeito indireto, no qual os taninos poderiam atuar indiretamente, melhorando a utilização proteica pelo hospedeiro e consequentemente uma melhor resposta imunológica deste aos parasitos (BUTTER et al., 2000; STRAIN; STEAR, 2001). Entretanto, o exato mecanismo de ação é incerto e pode diferir dependendo do parasito e estágio de desenvolvimento da planta (MIN; HART, 2003).

Os TC ligam-se às proteínas da dieta formando complexos (tanino-proteína), fazendo com que as proteínas de maior valor biológico não sejam degradadas e utilizadas pela microbiota ruminal, 
sendo estes complexos dissociados no intestino delgado, local de absorção dos aminoácidos (MIN et al., 2003; WAGHORN, 2008).

Forrageiras com alto teor de TC, quando fornecidos a ovinos e bovinos, melhoram a digestão proteica e absorção dos aminoácidos. Animais que apresentam uma maior absorção proteica produzem mais lã, carne e leite, assim como melhora na taxa de ovulação das fêmeas. $\mathrm{O}$ estado nutricional do animal é considerado um importante fator de equilíbrio na relação parasito-hospedeiro, assim como na patogênese da infecção parasitária (VALDERRÁBANO; DELFA; URIARTE, 2002).

Asuplementação dos ovinos com TC, oriundos de plantas forrageiras vem sendo utilizado no controle de nematódeos gastrintestinais de ovinos (IQBAL et al., 2007). TC podem ser utilizados no bloqueio do ciclo de nematódeos em pastagens contaminadas, desenvolvimento larval, reinfecções e reduzindo a viabilidade dos nematódeos (MOLAN et al., 2003).

\section{Avaliação da propriedade anti-helmíntica de plantas taniníferas}

Avaliando o efeito da forrageira Sulla (Hedysarum coronarium), em ovinos naturalmente infectados por nematódeos gastrintestinais, Niezen et al. (1995) relatam redução na contagem de OPG. No mesmo trabalho, animais experimentalmente infectados com $T$. colubriformis apresentaram redução no OPG e diminuição da carga parasitária. Em outro experimento, ovelhas que foram alimentadas com $H$. coronarium, que possui alta concentração de $\mathrm{TC}$, apresentaram redução no número de T. colubriformis no intestino (NIEZEN et al., 2002).

O resultado da suplementação com TC oriundo de sete plantas foi avaliado em larvas de T. colubriformis por Molan et al. (2000), onde relataram que todos os extratos apresentaram efeito inibitório na migração larval. Em outro estudo, Niezen et al. (1998) analisaram o efeito das plantas taniníferas Sulla e dois cultivares de Cornichão (H. coronarium, Lotus corniculatus e Lotus pedunculatus) e encontraram redução na carga parasitária e na contagem de OPG, concluindo que essas forrageiras podem ser incorporadas à alimentação de ovinos para reduzir a dependência das drogas anti-helmínticas.

Butter et al. (2000) avaliaram como fonte de TC o extrato da planta Schinopsis spp., comumente conhecido como extrato de quebracho (EQ), adicionados na dieta de ovinos com diferentes níveis de proteína, concluíram que a inclusão do EQ na dieta com baixos índices proteicos, acarretou redução da infecção por T. colubriformis baseado na redução da contagem de OPG de cordeiros.

Athanasiadou et al. (2000a) relatam que cordeiros infectados por T. colubriformis que receberam uma dieta contendo $8 \%$ de EQ por sete dias consecutivos, apresentaram redução da carga parasitária e da contagem de OPG. Em outro estudo, Athanasiadou et al. (2000b) avaliou a ação do EQ através do fornecimento de duas dietas, uma contendo 30 gramas de EQ e outra contendo $60 \mathrm{~g}$ de EQ por kg de matéria fresca, durante 10 semanas. Os autores relataram redução na carga parasitária e na fecundidade de T. colubriformis, independente da concentração do EQ. De acordo com os autores, o fornecimento de EQ reduz a taxa de estabelecimento e o desenvolvimento da infecção parasitária.

Marley et al. (2003) fornecendo dieta a base de Cornichão (L. corniculatus) e Chicória (Cichorium intybus) para cordeiros naturalmente infectados, relatam redução na carga parasitária e na contagem de OPG, em animais alimentados com L. corniculatus, não ocorrendo redução significativa quando alimentados com C. intybus. Por outro lado, Tzamaloukas et al. (2005) relataram que uma dieta baseada no fornecimento de C. intybus acarretou diminuição na carga parasitária de T.circumcincta e redução na implantação de larvas em ovinos, portanto, concluíram que o $C$. intybus pode ser uma espécie promissora no controle da carga parasitária de $T$. circumcincta em ovinos. 
A ação in vitro no desembainhamento de $\mathrm{L}_{3}$ de $H$. contortus e $T$. colubriformis tratadas com quatro extratos de plantas taniníferas (Castanheira: Castanea sativa, Pinheiro-da-Escócia: Pinus sylvestris, Érica: Erica erigena e Vassoura escocesa: Sarothamnus scoparius), foi avaliada por Bahuaud et al. (2006). De acordo com os autores os taninos estão envolvidos no processo inibitório de desembainhamento de larvas infectantes, visto que ocorreu redução significativa na taxa de desembainhamento de $\mathrm{L}_{3}$ de $H$. contortus frente às plantas $C$. sativa, $P$. sylvestris e E. erigena, enquanto larvas de $T$. colubriformis apresentaram redução da taxa de desembainhamento somente para as plantas C. sativa e P. sylvestris. Em estudos realizados com monômeros de TC em larvas de $H$. contortus e T. colubriformis, Brunet e Hoste (2006) relatam que monômeros de prodelfinidinas são mais potentes que os monômeros de procianidinas no desembainhamento de larvas de terceiro estagio.

Brunet et al. (2007) avaliaram a ação de diferentes concentrações de extrato de "Sainfoin" (Onobrychis viciifolia) em larvas de $H$. contortus e T. colubriformis. No teste in vitro as concentrações testadas foram de 150, 300, 600 e $1200 \mu \mathrm{g} \mathrm{mL}^{-1}$. Na

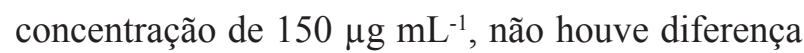
significativa na taxa de desembainhamento quando comparado com o controle negativo. A partir de $300 \mu \mathrm{g} \mathrm{mL} \mathrm{m}^{-1}$ ocorreu diferença significativa na taxa de eclosão para ambas as espécies de nematódeos. Com $1200 \mu \mathrm{g} \mathrm{mL}^{-1}$, observou-se inibição de $100 \%$ na taxa de desembainhamento das larvas de $T$. colubriformis e de $83 \%$ das larvas de $H$. contortus. No teste in vivo, animais receberam dieta com concentrações de 25,75 e $100 \%$ de extrato na dieta. Através de cânulas, larvas de $H$. contortus foram colocadas em contato com líquido ruminal por 160 minutos, sendo então observada diferença significativa na taxa de desembainhamento nas concentrações de $75 \%$ e $100 \%$. De acordo com os autores, ocorreu similaridade de ação entre os testes in vitro e in vivo.

As propriedades anti-helmínticas de quatro plantas taniníferas foram testadas por Alonso-Díaz et al. (2011). Utilizando extratos da leguminosa Havardia albicans e da árvore acácia (Acacia gaumeri) no teste in vitro de IML acarretou redução na taxa de migração de $H$. contortus para ambos os extratos, fato esse não observado quando se utilizou extratos de Castanha-maia (Brosimum alicastrum) e Leucena (L. leucocephala). No teste de desembainhamento, na dose de $75 \mu \mathrm{g} \mathrm{mL}$ 1, com os extratos de A. gaumeri e H. albicans, média de desembainhamento foi de $28,6 \%$ e $10,4 \%$ respectivamente, enquanto $B$. alicastrum e $L$. leucocephala apresentaram média de 97,5 e 93,3\%, respectivamente. Os autores relatam que os taninos são potentes inibidores de desembainhamento de L3 e de sua motilidade.

$\mathrm{O}$ efeito anti-helmíntico do extrato de quatro plantas taniníferas foi avaliado utilizando-se o teste de inibição da migração larval (IML) e o teste de desembainhamento (ALONSO-DÍAZ et al., 2008). Neste trabalho relataram que o extrato de Acácia (Acacia pennatula), Tamarindo selvagem (Lisyloma latisiliquum) e Leucena (Leucaena leucocephala) reduziu a taxa de migração das $\mathrm{L}_{3}$ e a utilização da Piscidia piscipula não acarretou inibição da migração. No teste de desembainhamento, utilizando as mesmas plantas, a redução na taxa de desembainhamento foi significativa para todas as plantas, confirmando o que o efeito anti-helmíntico dessas plantas contra $H$. contortus. Azando et al. (2011) avaliaram o efeito dos extratos de Árvora da fronteira africana (Newbouldia laevis) e "Candletree"(Zanthoxylum zanthoxyloides) como fontes de taninos. No teste in vitro de desembainhamento larval observaram que na concentração de $1200 \mu \mathrm{g} \mathrm{mL}-1$ de extrato, as taxas de desembainhamento de $H$. contortus foram de 5,19 e $0,93 \%$ para os extratos de $N$. laevis e $Z$. zanthoxyloides, respectivamente. Utilizando larvas de T. colubriformis as taxas foram de 4,22 e $0,72 \%$ para os extratos de $N$. laevis e Z. zanthoxyloides, respectivamente.

Manolarakiet al. (2010) testaram a ação anti- 
helmíntica dos TC de Lentisco (Pistacia lentiscus), Pinheiro-carrasco (Quercuscoccifera), Alfarrobeira (Ceratonia siliqua), Castanheiro (Castanea sativa), Pereira selvagem (Pyrus spinosa), "Sainfoin" (Onobrychis viciifolia) e Oliveira (Olea europaea), originárias da região Mediterrânea, observaram a IML de $H$. contortus, exceto para o extrato de $O$. europaea. No teste in vivo, ovinos infectados com $H$. contortus e $T$. colubriformis receberam P. lentiscus, $Q$. coccifera e $C$. siliqua na forma de farinha dos frutos incorporados à ração e $O$. viciifolia na forma de feno. Foi observada redução na eliminação de ovos, acarretado, principalmente, pela diminuição na fecundidade das fêmeas de ambas as espécies, uma vez que não foi relatada diminuição na carga parasitária dos animais.

Efeitos direto e indireto de TC em ovinos foram avaliados por Iqbal et al. (2007). No teste in vitro realizado com fonte de tanino comercial, ocorreu inibição da eclosão de ovos, entretanto, não foi efetivo em adultos de $H$. contortus. $\mathrm{O}$ teste in vivo indicou redução na contagem de OPG do grupo tratado com TC quando comparado ao controle não tratado. $\mathrm{O}$ efeito anti-helmíntico direto de TC foi evidenciado pela inibição da eclosão de ovos, portanto, a redução no OPG em ovinos pode validar a utilização de TC na alimentação de ovinos.

$\mathrm{Na}$ avaliação da ação da planta $L$. latisiliquum em ovinos experimentalmente infectados com H. contortus, Martínez-Ortíz-de-Montellano et al. (2010) relataram que o consumo em um curto período dessa planta na forma de forragem, altera a fecundidade das fêmeas e também diminui a eliminação de ovos nas fezes, mas não reduz a carga parasitária nos animais. De acordo com os autores a redução do número de OPG permite a menor contaminação das pastagens. Por outro lado, Max (2010) avaliou a ação dos TC extraído de Acacia mearnsii em ovinos naturalmente infectados e relataram reduções na eliminação de ovos e na carga parasitária.

Von Son-de Fernex et al. (2012) avaliaram a ação anti-helmíntica de cinco plantas taniníferas e observaram que Amendoim-forrageiro (Arachis pintoi), e as forrageiras Cratylia argentea, Cratylia argentea Yacapani e Cratylia argentea Veranera inibiram $100 \%$ o desembainhamento de larvas de $H$. contortus. No teste de inibição da migração larval, C. argentea, C.a. Veranera e Gliricidia sepium demonstraram ação anti-helmíntica inibindo a migração de larvas de $H$. contortus.

A ação anti-helmíntica de Lantana (Lantana Camara) Palo santo (Alpinia zerumbet), Cravode-defunto (Tagetes minuta) e Hortelã (Mentha villosa) foram avaliadas por Macedo et al. (2012), onde demonstraram que Alpinia zerumbet, Tagetes minuta e Mentha villosa, na concentração de $2,5 \mathrm{mg} \mathrm{mL} \mathrm{mL}^{-1}$, inibiram $82,7 \%, 96,8 \%$ e $97,6 \%$ respectivamente, a eclosão de ovos de $H$. contortus. No teste de desembainhamento ocorre inibição de $100 \%$ para todas as plantas testadas. De acordo com os autores, as plantas testadas podem ser utilizadas no controle de nematódeos gastrintestinais.

\section{Utilização de fontes taniníferas no Brasil}

No Brasil, têm sido realizados testes de eficácia anti-helmíntica com extratos de plantas como fonte de taninos. Pesquisa com o extrato de Acacia mearnsii (acácia negra) como fonte de TC na dose de 1,6 $\mathrm{g} \mathrm{kg}^{-1}$ PV, Minho (2006) descreveu uma redução na contagem de OPG de $H$. contortus e $T$. colubriformis e redução na carga parasitária de $H$. contortus. De acordo com o autor uso dos TC no controle alternativo da hemoncose ovina deve ser estudado e testado em regiões com problemas de resistência às drogas anti-helmínticas ou em fazendas que incorporam o sistema de produção orgânica. Em outro estudo utilizando também o extrato de A. mearnsii, Minho et al. (2008a) avaliaram os efeitos inibitórios do extrato sobre a alimentação de larvas de primeiro estágio $\left(\mathrm{L}_{1}\right)$ de $H$. contortus, $T$. colubriformis e T. circumcincta. Neste experimento foi relato que uma dose igual ou superior a $0,155 \mu \mathrm{g}$ $\mathrm{mL}^{-1}$ do extrato acarretou efeitos deletérios em $\mathrm{L}_{1}$ de 
todos os nematódeos avaliados, sendo que à partir dessa concentração, aproximadamente, $100 \%$ das larvas apresentaram-se inviáveis. A dose de $0,08 \mu \mathrm{g}$ $\mathrm{mL}^{-1}$ inativou aproximadamente $90 \%$ das $\mathrm{L}_{1}$. A dose descrita sugere a potencial utilização dessa fonte de TC no controle alternativo de parasitos.

Cenci et al. (2007) avaliaram os efeitos de TC de A. mearnsii em ovinos naturalmente infectados com helmintos gastrintestinais e observaram que ocorreu uma queda na contagem de OPG, indicando que o tanino da acácia apresenta eficácia anti-helmíntica, representando uma alternativa para o controle de helmintos em ovinos.A ação anti-helmíntica da A. Mearnsii também foi avaliada por Yoshihara (2012), utilizando-se o teste de inibição da eclosão de ovos e inibição da migração larval. De acordo com o autor, o extrato de acácia reduziu a eclosão de ovos e a migração das $\mathrm{L}_{3}$, demonstrando que o extrato tem ação ovicida e larvicida.

Minho et al. (2008b), analisaram o efeito dos TC provenientes do extrato de Acacia molissima em cordeiros naturalmente infectados com $H$. contortus e $T$. colubriformis relataram que houve redução na contagem de $\mathrm{OPG}$ e na carga parasitária de $H$. contortus no abomaso quando os mesmos receberam 1,6 $\mathrm{g} \mathrm{kg}^{-1} \mathrm{PV}$ de extrato, fato esse não observado na carga parasitária de $T$. colubriformis em intestino delgado.

Atividade ovicida e larvicida foi avaliada em H. contortus por Maciel et al. (2006) utilizando extrato de Melia azedarach (cinamomo). No teste de inibição da eclosão de ovos (IEO) utilizando extrato etanólico da folha observou-se que a mesma apresenta atividade ovicida, o que não foi observado quando se utiliza extrato hexânico da folha. Avaliando os extratos hexânico e etanólico das sementes observaram que os mesmos também apresentam atividade ovicida. No teste de inibição do desenvolvimento larval (TDL) ocorreu efeito inibitório significativo utilizando o extrato etanólico das folhas e o extrato clorofórmio das sementes. Baseado nos resultados, os autores afirmam que folhas e frutos de $M$. azedarach contêm compostos bioativos com ação anti-helmíntica contra $H$. contortus.

A ação ovicida e larvicida de extrato de Azadirachta indica (nim) em larvas de $H$. contortus foi avaliado por Costa et al. (2008), demonstrando que a inibição foi de $99,77 \%$ na eclosão na concentração de $3,12 \mu \mathrm{g} \mathrm{mL}^{-1}$ e inibição no desenvolvimento larvar de $87,11 \%$ na concentração de $50 \mathrm{mg} \mathrm{mL}^{-1}$ quando utilizou o extrato etanólico. $\mathrm{O}$ extrato acetato de etila na concentração de $50 \mu \mathrm{g}$ $\mathrm{mL}^{-1}$ a inibiu $51,31 \%$ na eclosão e $68,10 \%$ no teste de desembainhamento. Os autores verificaram que o extrato etanólico foi mais efetivo, sugerindo que o extrato de $A$. indica pode ser usado no controle de nematódeos gastrintestinais de pequenos ruminantes.

Oliveira et al. (2011) avaliaram a eficácia in vitro de Myracrodrum urundeuva (aroeira) contra H. contortus e relataram que o extrato de folhas mostrou-se efetivo, inibindo 97,73\% da eclosão na concentração de $1,25 \mathrm{mg} \mathrm{mL}^{-1}$, enquanto o extrato do caule inibiu a eclosão em $83,56 \%$ na concentração de $5 \mathrm{mg} \mathrm{mL}^{-1}$. Ao utilizar o extrato de folhas e caule no teste de desembainhamento na concentração de $0,31 \mathrm{mg} \mathrm{mL}-1$ ocorreu um bloqueio de 100\% no processo. De acordo com os autores, M. urundeuva pode ser usado para o controle de nematódeos gastrintestinais de pequenos ruminantes e a atividade anti-helmíntica da planta é, provavelmente, devido à presença de taninos.

\section{Ação direta dos TC sobre o parasito}

A redução do OPG em animais que recebem fontes de TC pode ser consequência de redução da carga parasitária, ou por diminuição da fecundidade das fêmeas (MARTÍNEZ-ORTÍZDE-MONTELLANO et al., 2010; MINHO et al., 2008b). Segundo Hoste et al. (2006) taninos podem afetar os processos biológicos dos nematódeos dependendo de onde e como os taninos se ligam com várias estruturas dos nematódeos tais como a 
bainha, cutícula, sistema digestivo ou reprodutivo. A diferença de susceptibilidade de larvas de $H$. contortus e T. colubriformis frente aos diferentes monômeros de taninos condensados pode estar relacionada à espécie de parasito e a composição da bainha e cutícula dos nematódeos (BRUNET; HOSTE, 2006). A habilidade dos TC se ligarem às proteínas e alterar as propriedades físicas e químicas das mesmas devem ser considerados, especialmente porque a bainha e a cutícula dos nematódeos são compostas de prolina e hidroxiprolina (FETTERER; RHOADS, 1993).

Helmintos parasitos de vida livre ou não, são limitados por uma cutícula verdadeira de material extracelular em forma de ligações cruzadas de colágenos e proteínas insolúveis sintetizadas e secretadas pela hipoderme. A estrutura pode ser relativamente simples, ou muito complexa, variando de um gênero para outro, mostrando diferenças regionais na estrutura dependendo da espécie (HALTON, 2004). A cutícula dos nematódeos é metabolicamente ativa e morfologicamente especializada para absorção seletiva de nutrientes e osmorregulação. Dessa maneira, a difusão passiva de anti-helmínticos pela cutícula pode, provavelmente, ser responsável pela deformação e destruição da superfície corporal dos nematódeos (ALVAREZ; MOTTIER; LANNSSE, 2007).

Desde a década de 70, a microscopia eletrônica (ME) vem sendo utilizada como ferramenta para estudo de taxonomia e o efeito de fármacos em cestódeos e nematódeos. Hoste et al. (2006) descreveram alterações na superfície da cutícula de T. colubriformis, com presença de rugas transversais após o contato do parasito com TC. Segundo os autores, a capacidade dos TC se ligarem às proteínas pode explicar as lesões observadas na cutícula pela microscopia eletrônica de varredura (MEV).

Brunet, Foruquaux e Hoste (2011) comparam a ultraestrutura de larvas de terceiro estágio com bainha e sem bainha de $H$. contortus e $T$. colubriformis, as quais foram colocadas em contato com extrato de $O$. viciifolia. Neste trabalho foram observadas alterações na camada hipodérmica, presença de numerosas vesículas no citoplasma e degeneração das células musculares e intestinais, resultando na morte larval. A frequência das lesões foi diferente, sendo que as alterações superficiais (camada hipodérmica e células musculares) foram mais frequentes em larvas embainhadas, enquanto que as larvas desembainhadas apresentaram maior incidência de alterações nas camadas mais profundas (células intestinais). Essas diferenças sugerem que as ações do extrato em diferentes tipos celulares dependem da presença ou não da bainha. Nas larvas embainhadas, as lesões podem estar associadas com a cutícula, provavelmente devido a um bloqueio de trocas através da cutícula com o meio ambiente, podendo resultar em asfixia ou toxicidade devido ao acúmulo de metabólitos do produto. As lesões das células intestinais das larvas desembainhadas podem ser em decorrência da ingestão dos metabólitos presentes no extrato, podendo interferir com os processos de digestão e afetar a nutrição. Esse estudo mostrou que a ultraestrutura de larvas dos nematódeos gastrintestinais pode ser alterada após o contato in vitro com extratos de plantas.

O efeito direto (in vitro e in vivo) do extrato de $O$. viciifolia e L. latisiliquum foi avaliado por MartínezOrtiz-de-Montellano et al. (2013) utilizando a MEV. No teste in vitro foram observadas presença de rugas longitudinais e transversais na cutícula ao longo do corpo e região cefálica de fêmeas de $H$. contortus quando em contato com o extrato. Também foram observados agregados dos extratos ao redor da cápsula bucal, região da vulva e ânus. No estudo in vivo, alterações semelhantes foram observadas, com exceção dos agregados que só foram descritos na região ao redor da cápsula bucal. De acordo com o autor, as alterações estruturais nos parasitos expostos aos extratos das plantas podem afetar a motilidade e nutrição com possíveis consequências na reprodução.

Para avaliar o efeito direto do extrato de $A$. mearnsii sobre nematódeos, Yoshihara (2012) 
utilizou testes in vitro e in vivo e detectou o efeito dos TC utilizando MEV. No teste in vitro foi observada a presença de rupturas da cutícula, com aumento de volume que distorcem as estriações cuticulares, com aparente extravasamento de material interno e presença de rugas transversais na cutícula em toda a extensão corporal. No teste in vivo foram observadas rupturas da cutícula, notando-se aumento de volume que distorcem as estriações cuticulares e aparentemente extravasamento de material interno, alterações estas semelhantes às observadas nas condições in vitro. Na região cefálica foram observadas alterações estruturais com perda da integridade.

Os efeitos anti-helmínticos das plantas ricas em TC podem ser atribuídos a diferentes mecanismos: redução na contagem de OPG, inibição da eclosão, inibição da alimentação larval, inibição do desenvolvimento larval, inibição da motilidade de $\mathrm{L}_{3}$ e inibição da sobrevivência e redução na carga parasitária de NGI adultos (MINHO et al., 2008a; COSTA et al., 2008; MARTÍNEZ-ORTÍZ-DEMONTELLANO et al., 2010; AZANDO et al., 2011; DASGUPTA; ROY; TANDON, 2010).

Diversos trabalhos relatam o potencial promissor de plantas ricas em taninos no controle de nematódeos gastrintestinais de ruminantes, sendo que esse metabólito está presente em muitas plantas nativas do Brasil, indicando um amplo campo de pesquisa de fitoterápicos com ação anti-helmíntica.

\section{Considerações Finais}

$\mathrm{O}$ interesse no efeito dos TC sobre ruminantes traz grandes perspectivas para o controle de nematódeos e redução na ocorrência de isolados resistentes às drogas anti-helmínticas, assim como um grande avanço e uma nova perspectiva para o desenvolvimento de sistemas de produção orgânica de ruminantes.

Além da possibilidade da utilização em sistemas de produção onde os quimioterápicos são restringidos ou proibidos, os TC podem ainda ser utilizados no controle alternativo, concomitantemente ao controle parasitário tradicional, com a finalidade de diminuir e espaçar as administrações das drogas anti-helmínticas convencionais.

O conhecimento da epidemiologia dos nematódeos é primordial, já que os TC agem na descontaminação das pastagens, diminuindo a incidência de manifestações clínicas da doença e, principalmente, diminuindo a pressão de seleção sobre os isolados de NGI. Com isso, podemos minimizar o aparecimento do fenômeno da multirresistência às drogas anti-helmínticas e, até mesmo, prolongar a eficácia das drogas em uso na atualidade e das que serão lançadas no futuro.

Estes resultados demonstram o potencial de utilização de plantas taniníferas no controle de nematódeos gastrintestinais de pequenos ruminantes. Portanto, a prospecção de compostos bioativos e o desenvolvimento de um produto antihelmíntico contendo TC teria um grande potencial comercial.

\section{Referências}

ALMEIDA, F. A.; GARCIA, K. C. O. D.; TORGERSON, P. R.; AMARANTE, A. F. T. Multiple resistance to anthelmintics by Haemonchus contortus and Trichostrongylus colubriformis in sheep in Brazil. Parasitology International, Kiyotake, v. 59, n. 4, p. 622625, 2010.

ALONSO-DÍAZ, M. A.; TORRES-ACOSTA, J. F. J.; SANDOVAL-CASTRO, C. A.; HOSTE, H. Comparing the sensitivity of two in vitro assays to evaluate the anthelmintic activity of tropical tannin rich plant extracts against Haemonchus contortus. Veterinary Parasitology, Amsterdam, v. 181, n. 2-4, p. 360-364, 2011.

ALONSO-DÍAZ, M. A.; TORRES-ACOSTA, J. F. J.; SANDOVAL-CASTRO, C. A.; AGUILARCABALlERO, A. J.; HOSTE, H. In vitro larval migration and kinetics of exsheathment of Haemonchus contortus larvae exposed to four tropical tanniniferous plant extracts. Veterinary Parasitology, Amsterdam, v. 153, n. 3-4, p. 313-319, 2008.

ALONSO-DÍAZ, M. A.; TORRES-ACOSTA, J. F. J.; SANDOVAL-CASTRO, C. A.; HOSTE, H. Tannins and 
tropical tree fodders fed to small ruminants: a friendly foe?. Small Ruminant Research, Amsterdam, v. 89, n. 2-3, p. 164-173, 2010.

ALVAREZ, L. L.; MOTTIER, M. L.; LANNSSE, C. E. Drugs transfer into target helminth parasites. Trends in Parasitology, Philadelphia, v. 23, n. 3, p. 97-104, 2007.

ALVAREZ-SÁNCHEZ, M. A.; PÉREZ-GARCIA, J.; CRUZ-ROJO, M. A.; ROJO-VÁZQUEZ, F. A. Anthelmintic resistance in trichostrongylid nematodes of sheep farms in Northwest Spain. Parasitology Research, Berlin, v. 99, n. 1, p. 78-83, 2006.

AMARANTE, A. F. T. Controle de endoparasitoses dos ovinos. In: Sociedade Brasileira de Zootecnia. A produção animal na visão dos brasileiros. Piracicaba: FEALQ, 2001. p. 461-473.

AMARANTE, A. F. T.; BAGNOL JUNIOR, J.; AMARANTE, M. R. V.; BARBOSA, M. A. Host specificity of sheep and cattle nematodes in São Paulo state, Brazil. Veterinary Parasitology, Amsterdam, v. 73, n. 1-2, p. 89-104, 1997.

AMARANTE, A. F. T.; BRICARELLO, P. A.; ROCHA, R. A.; GENNARI, S. M. Resistance of Santa Ines, Suffolk and Ile de France lambs to naturally acquired gastrointestinal nematode infections. Veterinary Parasitology, Amsterdam, v. 120, n. 1-2, p. 91-106, 2004.

ATHANASIADOU, S.; KYRIAZAKIS, I.; JACKSON, S.; COOP, R. L. Consequences of long-term feeding with condensed tannins on sheep parasited with Trichostrongylus colubriformis. International Journal for Parasitology, Oxford, v. 30, n. 9, p. 1025-1033, 2000b.

Direct anthelmintic effect of condensed tannins towards different gastrointestinal nematodes of sheep: In vitro and in vivo studies. Veterinary Parasitology, Amsterdam, v. 99, n. 3, p. 205-219, 2001.

Effects of short-term exposure to condensed tannins on adult Trichostrongylus colubriformis. Veterinary Record, London, v. 146, n. 25, p. 728-732, 2000a.

AZANDO, E. V. B.; HOUNZANGBÉ-ADOTÉ, M. S.; OLOUNLADE, P. A.; BRUNET, S.; FABRE, N.; VALENTIN, A.; HOSTE, H. Involvement of tannins and flavonoids in the in vitro effects of Newbouldia laevis and Zanthoxylum zanthoxyloides extracts on the exsheathment of third-stage infective larvae of gastrointestinal nematodes. Veterinary Parasitology, Amsterdam, v. 180, n. 3-4, p. 292-297, 2011.

BAHUAUD, D.; MARTINEZÓRTIZ DE MONTELLANO, C.; CHAUVEAU, S.; PREVOT, F.; TORRES-ACOSTA, F.; FOURASTE, I.; HOSTE, H.
Effects of four tanniferous plant extracts on the in vitro exsheathment of third-stage of parasitic nematodes. Parasitology, Cambridge, v. 132, n. 4, p. 545-554, 2006.

BAR-NUM, N.; MAYER, A. M. Cucurbitacins protect cucumber tissue against infection by Botrytis cinera. Phytochemistry, Oxford, v. 29, n. 3, p. 787-791,1990.

BARRY, T. N.; McNABB, W. C. The implication of condensed tannins on the nutritive value of temperature forages fed to ruminants. British Journal of Nutrition, Cambridge, v. 81, n. 4, p. 263-272, 1999.

BARTLEY, D. J.; JACKSON, E.; JOHNSTON, K.; COOP, R. L.; MITCHELL, G. B. B.; SALES, J.; JACKSON, F. A survey of anthelmintic resistant nematode parasites in Scottish flocks. Veterinary Parasitology, Amsterdam, v. 117, n. 1-2, p. 61-71, 2003.

BRUNET, S.; AUFRERE, J.; BABILI, F.; FOURASTE, I.; HOSTE, H. The kinetics of exsheathment of infective nematode larvae is disturbed in presence of a tanninrich plant extract (sainfoin) both in vitro and in vivo. Parasitology, Cambridge, v. 134, n. 9, p. 1253-1262, 2007.

BRUNET, S.; FORUQUAUX, I.; HOSTE, H. Ultrastructural changes in the third-stage, infective larvae of ruminant nematodes treated with sainfoin (Onobrychis viciifolia) extract. Parasitology International, Kiyotake, v. 60, n. 4, p. 419-424, 2011.

BRUNET, S.; HOSTE, H. Monomers of condensed tannins affect the larval exsheathment of parasitic nematodes of ruminats. Journal of Agricultural and Food Chemistry, Washington, v. 54, n. 20, p. 7481-7487, 2006.

BUTTER, N. L.; DAWSON, J. M.; WAKELIN, D.; BUTTERY, P. J. Effect of dietary tannin and protein concentration on nematode infection (Trichostrongylus colubriformis) in lambs. Journal of Agricultural Science, Cambridge, v. 134, n. 1, p. 89-99, 2000.

CENCI, F. B.; LOUVANDINI, H.; MCMANUS, C. M.; DELL'PORTO, A.; COSTA, D. M.; ARAÚJO, S. C.; MINHO, A. P.; ABDALLA, A. L. Effects of condensed tannin from Acacia mearnsii on sheep infected naturally with gastrointestinal helminthes. Veterinary Parasitology, Amsterdam, v. 144, n. 1-2, p. 132-137, 2007.

CEZAR, A. S.; TOSCAN, G.; CAMILLO, G.; SANGIONI, L. A.; RIBAS, H. O.; VOGEL, F. S. F. Multiple resistance of gastrointestinal nematodes to nine different drugs in sheep flock in southern Brazil. Veterinary Parasitology, Amsterdam, v. 173, n. 1-2, p. 157-160, 2010.

COSTA, C. T. C.; BEVILAQUA, C. M. L.; CAMURÇAVASCONCELOS, A. L. F.; MACIEL, M. V.; MORAIS, S. M.; CASTRO, C. M. S.; BRAGA, R. R.; OLIVEIRA, 
L. M. B. In vitro ovicidal larvicidal activity of Azadiractha indica extracts on Haemonchus contortus. Small Ruminant Research, Amsterdam, v. 74, n. 1-3, p. 284-287, 2008.

DASGUPTA, S.; ROY, B.; TANDON, V. Ultrastructural alterations of the tegument of Raillietina echinobothrida treated with the stem bark of Acacia oxyphylla (Leguminosae). Journal of Ethnopharmacology, Limerick, v. 127, n. 2, p. 568-571, 2010.

DOS SANTOS, V. T.; GONÇALVES, P. C. Verificação de estirpe resistente de Haemonchus resistente ao thiabendazole no Rio Grande do Sul (Brasil). Revista da Faculdade de Agronomia e Veterinária, Porto Alegre, v. 9, n. 1, p. 201-209, 1967.

DRUDGE, J. H.; SZANTO, J.; WYATT, Z. N.; ELAM, G. Field studies on parasite control in sheep: Comparison of thiabendazole, ruelene, and phenothiazine. American Journal of Veterinary Research, Chicago, v. 25, n. 1, p. 1512-1518. 1964.

ECHEVARRIA, F. A. M.; PINHEIRO, A. C.; CORRÊA, M. B. C. Controle estratégico da verminose ovina no Rio Grande do Sul. Bagé: CBPV, 1989. p. 159-163. (Curso de parasitologia animal, 2).

FETTERER, R. H.; RHOADS, M. L. Biochemistry of the nematode cuticle: relevance to parasitic nematodes of livestock. Veterinary Parasitology, Amsterdam, v. 46, n. 1-4, p. 103-111, 1993.

FIEL, C.; GUZMAN, M.; STEFFAN, P.; RODRIGUEZ, E.; PRIETO, O.; BHUSHAN, C. The efficacy of Trichlorphon and Naphthalophos against multiple anthelmintic resistant nematodes of naturally infect sheep in Argentina. Parasitology Research, Berlin, v. 109, n. 1, p. S139-S148, 2011.

GEORGE, N.; PERSAD, K.; SAGAM, R.; OFFIAH, V. N.; ADESIYUN, A. A.; HAREWOOD, W. Efficacy of commonly used anthelmintics: first report of multiple drug resistance in gastrointestinal nematodes of sheep in Trinidad. Veterinary Parasitology, Amsterdam, v. 183, n. 1-2, p. 194-197, 2011.

GOPAL, R. M.; POMROY, W. E.; WEST, D. M. Resistance of field isolates of Trichostrongylus colubriformis and Ostertagia circumcincta to ivermectin. International Journal for Parasitology, Oxford, v. 29, n. 5, p. 781-786, 1999.

HALTON, D. Microscopy and the helminth parasite. Micron, Oxford, v. 35, n. 5, p. 361-390, 2004.

HAMMOND, J. A.; FIELDING, D.; BISHOP, S. C. Prospects for plant anthelmintics in tropical veterinary medicine. Veterinary Research Communications, Dordrecht, v. 21, n. 3, p. 213-228, 1997.
HOSTE, H.; JACKSON, F.; ATHANASIADOU, S.; THAMSBORG, S. M.; HOSKIN, S. O. The effects of tannin-rich plants on parasitic nematodes in ruminants. Trends in Parasitology, Philadelphia, v. 22, n. 6, p. 253261, 2006.

IQBAL, Z.; SARWAR, M.; JABBAR, A.; AHMED, S.; NISA, M.; SAJID, M. S.; KHAN, M. N.; MUFTI, K. A.; YASEEN, M. Direct and indirect anthelmintic effects of condensed tannins in sheep. Veterinary Parasitology, Amsterdam, v. 144, n. 1-2, p. 125-131, 2007.

JACKSON, F.; HOSTE, H. In vitro methods for primary screening of plant products direct activity against ruminant gastrointestinal nematodes. In: VERCOE, P. E.; MAKKAR, H. P. S.; SCHLINK, A. C. In vitro screening of plants resources for extra-nutritional attributes in ruminants: nuclear and related methodologies. London: Springer, 2010. p. 25-45.

JACKSON, F.; COOP, R. L. The development of anthelmintic resistance in sheep nematodes. Parasitology, Cambridge, v. 120, p. S95-S107, 2000. Supplement 1.

KAPLAN, R. M. Drug resistance in nematodes of veterinary importance: a status report. Trends in Parasitology, Philadelphia, v. 20, n. 10, p. 477-481, 2004.

KOHLER, P. The biochemical basis of anthelmintic action and resistance. International Journal for Parasitology, Oxford, v. 31, n. 4, p. 336-345, 2001.

MACEDO, I. T. F.; BEVILAQUA, C. M. L.; OLIVEIRA, L. M. B.; CAMURÇA-VASCONCELOS, A. L. F.; MORAIS, S. M.; MACHADO, L. K. A.; RIBEIRO, W. L. C. In vitro activity of Lantana camara, alpinia zerumbet, Mentha villosa and Tagetes minuta decoctions on Haemonchus contortus eggs and larvae. Veterinary Parasitology, Amsterdam, v. 190, n. 3-4, p. 504-509, 2012

MACIEL, M. V.; MORAIS, S. M.; BEVILAQUA, C. M. L.; CAMURÇA-VASCONCELOS, A. L. F.; COSTA, C. T. C.; CASTRO, C. M. S. Ovicidal and larvicidal activity of Melia Azedarach extracts on Haemonchus contortus. Veterinary Parasitology, Amsterdam, v. 140, n. 5, p. 98104, 2006.

MAKKAR, H. P. S.; FRANCIS, G.; BECKER, K. Bioactivity of phytochemicals in some lesser-know plants and their effects and potential applications in livestock and aquaculture production systems. Animal, Cambridge, v. 1, n. 9, p. 1371-1391, 2007.

MANOLARAKI, F.; SOTIRAKI, S.; STEFANAKIS, A.; SKAMPARDONIS, V.; HOSTE, M.; HPSTE, H. Anthelmintic activity of some Mediterranean browse plants against parasitic nematodes. Parasitology, Cambridge, v. 137, n. 4, p. 685-696, 2010. 
MARLEY, C. L.; COOK, R.; KEATINGE, R.; BARRET, J.; LAMPKIN, N. H. The effect of birdsfoot trefoil (Lotus corniculatus) and chicory (Cichorium intybus) on parasite intensities and performance of lambs naturally infected with helminth parasites. Veterinary Parasitology, Amsterdam, v. 112, n. 1-2, p. 147-155, 2003.

MARTÍNEZ-ORTIZ-DE-MONTELLANO, C.; ARROYO-LÓPEZ, C.; FOURQUAUX, I.; TORRESACOSTA, J. F. J.; SANDOVAL-CASTRO, C. A.; HOSTE, H. Scanning electron microscopy of Haemonchus contortus exposed to tannin-rich plants under in vivo and in vitro conditions. Experimental Parasitology, New York, v. 133, n. 3, p. 281-286, 2013.

MARTÍNEZ-ORTIZ-DE-MONTELLANO,

C.; VARGAS-MAGAÑA， J. J.; CANUL-KU, H. I.; MIRANDA-SOBERANIS, R.; CAPETILLO-LEAL, C.; SANDOVAL-CASTRO, C. A.; HOSTE, H.; TORRES-ACOSTA, J. F. J. Effect of a tropical tanninrich plant Lysiloma latisiliquum on adult populations of Haemonchus contortus in sheep. Veterinary Parasitology, Amsterdam, v. 172, n. 3-4, p. 283-290, 2010.

MATOS, A. F. J. Living pharmacies. Journal of the Brazilian Association for the Advancement of Science, São Paulo, v. 49, n. 5-6, p. 409-412, 1997.

MAX, R. A. Effect of repeated wattle tannin drench on worm burdens, fecal egg counts and egg hatchability naturally acquired nematode infections in sheep and goats. Veterinary Parasitology, Amsterdam, v. 169, n. 1-2, p. 138-143, 2010.

MELO, A. C. F. L.; REIS, I. F.; BEVILAQUA, C. M. L.; VIEIRA, L. S.; ECHEVARRIA, F. A.; MELO, L. M. Nematódeos resistentes a anti-helmínticos em rebanhos de ovinos e caprinos do estado do Ceará, Brasil. Ciência Rural, Santa Maria, v. 37, n. 2, p. 339-344, 2003.

MIN, B. R.; BARRY, T. N.; ATTWOOD, G. T.; McNABB, W. C. The effect of condensed tannins on the nutrition and health of ruminants fed fresh temperate forages: a review. Animal Feed Science and Technology, Washington, v. 106, n. 1-4, p. 3-19, 2003.

MIN, B. R.; HART, S. P. Tannin for suppression of internal parasites. Journal of Animal Science, Albany, v. 81, n. 14, E.102-E.109, 2003. Supplement 2.

MINHO, A .P. Efeito anti-helmíntico de taninos condensados sobre nematódeos gastrintestinais em ovinos. 2006. Tese (Doutorado em Ciências) - Centro de Energia Nuclear na Agricultura da Universidade de São Paulo, Piracicaba.

MINHO, A. P.; BUENO, I. C. S.; GENNARI, S. M.; JACKSON, F.; ABDALLA, A. L. In vitro effect of condensed tannin extract from Acacia (Acacia mearnsii) on gastrintestinal nematodes of sheep. Revista Brasileira de Parasitologia Veterinária, São Paulo, v. 17, p. 147151, 2008a. Supplement 1.

MINHO, A. P.; BUENO, I. C. S.; LOUVANDINI, H.; JACKSON, F.; GENNARI, S. M.; ABDALLA, A. L. Effect of Acacia molissima tannin extract on the control of gastrointestinal parasites in sheep. Animal Feed Science and Technology, Washington, v. 147, n. 1-3, p. 172-181, 2008b.

MOLAN, A. L.; MEAAGHER, L. P.; SPENCER, P. A.; SIKAKUMARAN, S. Effect of flavan-3-ols on in vitro egg hatching, larval development and viability of infective larvae of Trichostrongylus colubriformis. International Journal for Parasitology, Oxford, v. 33, n. 14, p. 1691-1698, 2003.

MOLAN, A. L.; WAGHORN, G. C.; MIN, B. R.; MCNABB, C. The effect of condensed tannins from seven herbages on Trichostrongylus colubriformis larval migration in vitro. Folia Parasitologica, Ceské Budejovice, v. 47, n. 1, p. 39-44, 2000.

MOLENTO, M. B.; PRICHARD, R. K. Nematode control and the possible development of anthelmintic resistance. Revista Brasileira de Parasitologia Veterinária, São Paulo, v. 8, n. 1, p. 75-86, 1999.

NIEZEN, J.H.;CHARLESTON,W.A.G.;ROBERTSON, H. A.; SHELTON, D.; WAGHORN, G. C.; GREEN, R. The effect of feeding sulla (Hedysarum coronarium) or lucerne (Medicago sativa) on lamb parasite burdens and development of immunity to gastrointestinal nematodes. Veterinary Parasitology, Amsterdam, v. 105, n. 3, p. 229245, 2002.

NIEZEN, J. H.; WAGHORN, T. S.; CHARLESTON, W. A. G.; WAGHORN, G. C. Growth and gastrointestinal parasitism in lambs grazing either lucerne (Mendicato sativa) or sulla (Hedysarum coronarium) which contains condensed tannins. Journal of Agricultural Science, Cambridge, v. 125, n. 2, p. 281-289, 1995.

NIEZEN, J. H.; CHARLESTON, W. A. G.; HODSON, J.; MACKAY, A. D.; LEATHWICK, D. M. Controlling internal parasites in grazing ruminants without recourse to anthelmintics: approaches, experiences and prospects. International Journal for Parasitology, Oxford, v. 26, n. 8-9, p. 983-992, 1996.

NIEZEN, J. H.; ROBERTSON, H. A.; WAGHORN, G. C.; CHARlESTON, W. A. Production, faecal egg counts and worm burdens of ewe lambs which grazed six contrasting forages. Veterinary Parasitology, Amsterdam, v. 80, n. 1, p. 15-27, 1998.

OLIVEIRA, L. M. B.; BEVILAQUA, C. M. L.; MACEDO, I. T. F.; MORAIS, S. M.; MACHADO, L. 
K. A.; CAMPELLO, C. C.; MESQUITA, M. A. Effects of Myracrodrum urundeuva extracts on egg hatching and larval exsheathment of Haemonchus contortus. Parasitology Research, Paris, v. 109, n. 3, p. 893-898, 2011.

RAMOS, C. I.; BELlATO, V.; ÁVILA, V. S.; COUTINHO, G. C.; SOUZA, A. P. Resistência de parasitos gastrintestinais de ovinos a alguns antihelmínticos no estado de Santa Catarina, Brasil. Ciência Rural, Santa Maria, v. 32, n. 3, p. 473-477, 2002.

RATES, S. M. K. Plants as source of drugs. Toxicon, Kidlington, v. 39, n. 5, p. 603-613, 2001.

SANGSTER, N. C.; GILL, J. Anthelmintic resistance: past, present and future. International Journal for Parasitology, Oxford, v. 29, n. 1, p. 115-124, 1999.

SCHNYDER, M.; TORGERSON, P. R.; SCHÖNMANN, M.; KOHLER, L.; HERTSBERG, H. Multiple anthelmintic resistance in Haemonchus contortus isolated from south African Boer goats in Switzerland. Veterinary Parasitology, Amsterdam, v. 128, n. 3-4, p. 185-190, 2005.

SCZESNY-MORAES, E. A.; BIANCHIN, I.; SILVA, K. F.; CATTO, J. B.; HONER, M. R.; PAIVA, F. Resistência anti-helmíntica de nematóides gastrintestinais em ovinos, Mato Grosso do Sul. Pesquisa Veterinária Brasileira, Brasilia, v. 30, n. 3, p. 229-236, 2010.

SOUTHERLAND, I. A.; DAMSTEEGT, A.; MILLER, C. M.; LEATHWICK, D. M. Multiple species of nematode resistant to ivermectin and benzimidazolelevamisole combination on a sheep farm in New Zealand. New Zealand Veterinary Journal, Wellington, v. 56, n. 2, p. 67-70, 2008.

SOUZA, F. P.; THOMAZ-SOCCOL, V. Contribuição para o estudo da resistência de helmintos gastrintestinais de ovinos (Ovis aries) aos anti-helmínticos, no estado do Paraná. Revista Brasileira de Parasitologia Veterinária, São Paulo, v. 6, n. 2, p. 217, 1997. Suplemento 1.

STRAIN, S. A. J.; STEAR, M. J. The influence of protein supplementation on the immune response to Haemonchus contortus. Parasite Immunology, Oxford, v. 23, n. 10, p. 527-531, 2001.

THOMAZ-SOCCOL, V.; SOUZA, F. P.; SOTOMAIOR, C.; CASTRO, E. A.; MILCZEWSKI, V.; MOCELIN, G.; SILVA, M. C. P. Resistance of gastrointestinal nematodes to anthelmintics in sheep (Ovis aires). Brazilian Archives of Biology and Technology, Curitiba, v. 47, n. 1, p. 41-47, 2004.
TORRES-ACOSTA, J. F. J.; HOSTE, H. Alternative or improved methods to limit gastrointestinal parasitism in grazing sheep and goats. Small Ruminant Research, Amsterdam, v. 77, n. 2-3, p. 159-173, 2008.

TZAMALOUKAS, O.; ATHANASIADOU, S.; KYRIAZAKIS, I.; JACKON, F.; COOP, R. L. The consequences of short-term grazing of bioactive forages on established adult and incoming larvae populations of Teladorsagia circumcincta in lambs. International Journal for Parasitology, Oxford, v. 35, n. 3, p. 329-335, 2005.

VALDERRÁBANO, J.; DELFA, R.; URIARTE, J. Effect of feed intake on the development of gastrointestinal parasitism in growing lambs. Veterinary Parasitology, Amsterdam, v. 104, n. 4, p. 327-338, 2002.

VIEIRA, L. S.; CAVALCANTE, A. C. R.; PEREIRA, M. F.; DANTAS, L. B.; XIMENES, L. J. F. Evaluation of anthelmintic efficacy of plants available in Ceará, North - East Brazil, for the control of goat gastrointestinal nematodes. Revue Medicine Veterinary, Toulosse, v. 150, n. 5, p. 447-452, 1999.

VON SON-DE FERNEX, E.; ALONSO-DIAZ, M. A.; VALLES-DE La MORA, B.; CAPETILLO-LEAL, C. M. In vitro anthelmintic activity of five tropical legumes on the exsheathment and motility of Haemonchus contortus infective larvae. Experimental Parasitology, New York, v. 131, n. 4, p. 413-418, 2012.

WAGHORN, G. Benefical and detrimental effects of dietary condensed tannins for sustainable sheep and goat production. Progress and Challenges. Animal Feed Science and technology, Washington, v. 174, n. 1-3, p. 116-139, 2008.

WALLER, P. J. Anthelmintic resistence. Veterinary Parasitology, Amsterdam, v. 72, n. 3-4, p. 391-412, 1997.

WARUIRU, R. M. Efficacy of closantel, albendazole and levamisole on an ivermectin resistant strain of Haemonchus contortus in sheep. Veterinary Parasitology, Amsterdam, v. 73, n. 1-2, p. 65-71, 1997.

YOSHIHARA, E. Efeito anti-helmíntico direto de taninos condensados em nematódeos gastrintestinais de ovinos (Ovis aries). 2012. Tese (Doutorado em Ciência Animal) - Universidade Estadual de Londrina, Londrina. 
